\title{
Decision analysis:
}

\section{A toy or a tool for clinical practice?}

$I^{2}$ their paper evaluating cefepime monotherapy, Halpern et al (pages 19-27) use a decision analysis modelling method to determine costs and outcomes associated with this therapy. Decision analysis has its origins in the disciplines of economics and management studies (1) and, over the past two decades, has been applied to many aspects of medical practice $(2,3)$. The main benefit of clinical decision analysis (CDA) is that it forces one to lay out and analyze critically approaches to therapy for a given disease state. One group has gone so far as to raise the methodology to the level of a clinical consultation service (5). Pharmacoeconomic evaluation is a more recent variation of $\mathrm{CDA}$ that has developed as we try to improve our decision making mechanisms.

By definition, decision analysis is a process; that is, a systematic approach to decision making under conditions of uncertainty (eg, where there is imperfect or incomplete information available) (5). It allows one to link choices, actions and outcomes in an effort to improve the decision made. The process, more commonly referred to as CDA modelling, is described by a series of fundamental stages: one identifies that there is a need to make a choice from among a number of options; each option leads to multiple consequences; the probability or uncertainty associated with each consequence is estimated in some manner; and one assesses the value attributable to each consequence (via costs, utilities or some measure of effectiveness).

$\mathrm{CDA}$ modelling involves pulling together related information from a variety of data sources to create a scenario reflecting the choice(s) which must be made and its (their) consequences. Where information is not specifically available, assumptions are made based on clinical experience, assumptions that are then subject to challenge during sensitivity analysis. Sensitivity analysis is an integral part of CDA, in that it tests the model for robustness (a reliability issue) and, some would argue, for validity. It is imperative that the estimates and/or assumptions used for key parameters in the model (especially those based on 'opinion' or where there is a high degree of uncertainty or variability) be subjected to sensitivity analysis.

One of the major advantages of CDA methodology is its ability to change decision making from a process that is subjective and nebulous into a concrete entity that is capable of identifying the decision option that should be chosen based on the information put into the model. The fundamental premise underlying decision analysis is the belief that the tradition- ally intuitive approach to decisions and judgements can be improved by a systematic and explicit analysis which yields a resolution based on quantitative methods. The explicit nature of the process can facilitate communication among those involved in or affected by the decision, with the opportunity to refine and improve the analytic approach. (CDA forces one to develop a graphic representation of the possible outcomes of the choices [ie, decision tree] that can be tested [via sensitivity analysis] to determine the validity of the model.) The process also forces decision makers to accept the uncertainty of the outcomes that result from their choices and to quantify them so as to be used as part of the decision process (6).

While one may understand what CDA can do, one also needs to understand the caveats and pitfalls that go hand in hand with the methodology. The greatest challenge to the validity of any CDA model is the quality of the information used in its development. There is a hierarchy of information used as the source of data put into the model (ie, randomized controlled trials [RCTs], other literature sources, reputable and validated databases, unpublished data, subjective information from clinicians, etc). While any or all of these sources might be used in a given analysis, it is the degree of reliance on each of the sources that must be considered when assessing model validity.

In addition to assessing the quality of information used in the model, the reader must make his or her own assessment of the model's face validity. This applies to the applicability of the information put into the model (let alone the scenario described by the decision tree) vis-à-vis one's local disease and practice patterns. The CDA method does not allow one to abdicate one's responsibility to challenge the model. One must use one's clinical knowledge to evaluate critically the assumptions that form the basis of the model: Do they reflect the way one practises, the patient population, or the way microorganisms behave in a particular institution or region? Are the alternatives included in the analysis reasonable? Is the model too simplistic in its description of the typical sequence of events and outcomes?

In their paper, Halpern et al have demonstrated some of both the benefits and pitfalls of the CDA method. The investigators were diligent in trying to obtain Canadian-based microbiological data because the frequency of infection caused by various organisms may differ significantly across borders. On the other hand, the use of multiple data sources for bacterial resistance rates may have introduced error into the model. The 
problem lies in the comparability of patient populations from which the resistant isolates were obtained. When reviewing a study, it behooves the reader to examine the original papers from which estimates are derived to ensure comparability to local conditions. On another note, the authors were not specific regarding the particular parameters evaluated in the sensitivity analysis. As a result, the degree to which variable resistance patterns affect the outcome of the model is unclear.

While most of the data used in this analysis came from secondary sources, all are reputable and validated sources of information. There was minimal reliance on expert opinion, except where no generalizable data were available (eg, choice of alternative antibiotics). This is acceptable methodology, although it introduces a factor that may form the basis of challenges to the model. While not an issue in this study, ethical concerns may also arise where expert opinion is used in model development. Readers should be able to ascertain from the study report whether clinical experts were paid for their information. Data from such sources may undermine the credibility of a model, no matter how rigorous the remaining information and methods used.

So how might this example of the use of CDA in decision making be applied in institutions? First, I would disagree that cefepime's prime advantage is in its ability to decrease hospital length of stay (LOS). As so nicely demonstrated in the threshold analysis, the increase in baseline LOS for all of the comparator agents is one half-day (or less). And this is where we bring clinical reality back into the decision making process. Given that LOS is the main cost driver in the model, can we really save those partial days of LOS in order to achieve any savings that might accrue from use of this medication? Many would say probably not, despite the improvements in efficiency that we have seen in the health care sector over the past five years or so. If cost is going to drive the decision of whether to use this new product, then perhaps the costs per patient demonstrated in Tables 6 and 7 should be the basis of any attempt to place this product in a microbiological niche on hospital formularies. These comments are not to say that the analysis by Halpern et al was in any way erroneous. They are merely meant to remind the reader not to take any analysis at face value and to alert the reader to the kinds of questions they should be asking themselves as they evaluate this and other analyses from their own perspective.

Proponents of CDA certainly do not intend that the health care community should do away with RCTs and other tools of evidence-based medicine. These more traditional and finely tuned methods should continue to be the gold standard by which we try to solve clinical dilemmas. However, RCTs do not exist for every therapeutic issue. Decision analysis offers an alternative approach to addressing clinical problems where clear-cut answers are not immediately apparent. $\mathrm{CDA}$ is a useful methodology for explicitly defining the decision making processes. However, those decisions cannot be made simply on the basis of a single published analysis. And if we do use these analyses to facilitate the process, we cannot abdicate our responsibility to appraise critically the CDA model for both its content and face validity.

\section{REFERENCES}

1. Barr JT, Schumacher GE. Decision analysis and pharmacoeconomic evaluations. In: Bootman JL, Townsend RJ, McGhan WF, eds. Principles of Pharmacoeconomics, 2nd edn. Cincinnati: Harvey Whitney Books Company, 1996.

2. Weinstein MC, Fineberg HV, eds. Clinical Decision Analysis. Philadephia: WB Saunders and Company, 1980.

3. Pauker SG, Kassierer JP. Decision analysis. N Engl J Med 1987;316:250-8

4. Plante DA, Kassirer JP, Zarin DA, Pauker SG. Clinical decision consultation service. Am J Med 1986;80:1169-76.

5. Barr JT, Schumacher GE. Applying decision analysis to pharmacy management and practice decisions. Top Hosp Pharm Manage 1994;13:60-71.

\author{
Judith L Glennie FCSHP \\ Pharmacoeconomics Consultant \\ Pharmaceutical Outcomes Research Unit \\ Ottawa Ceneral Hospital \\ Ottawa, Ontario
}




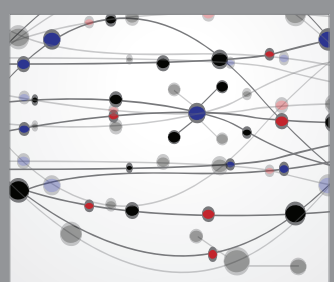

The Scientific World Journal
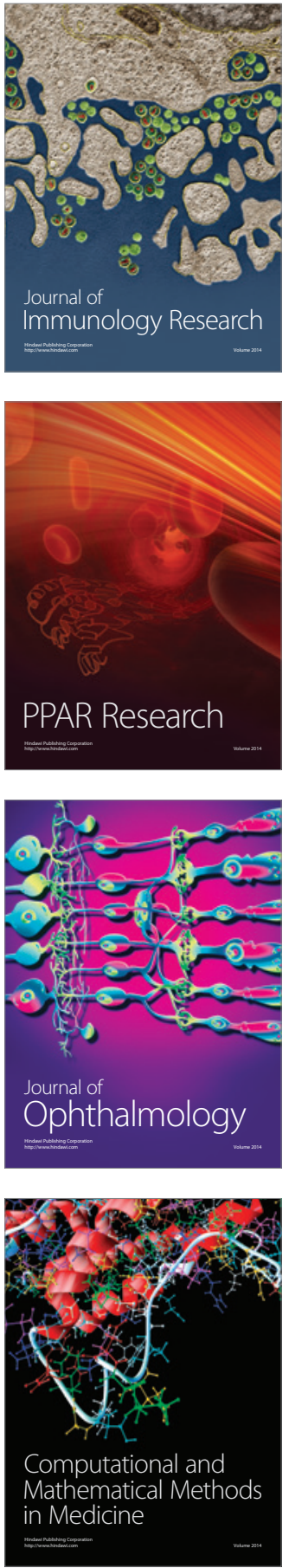

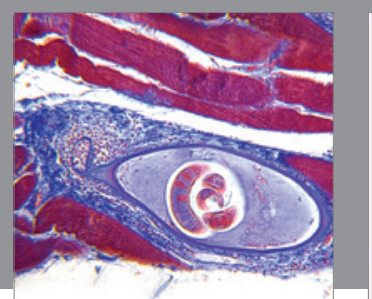

Gastroenterology Research and Practice

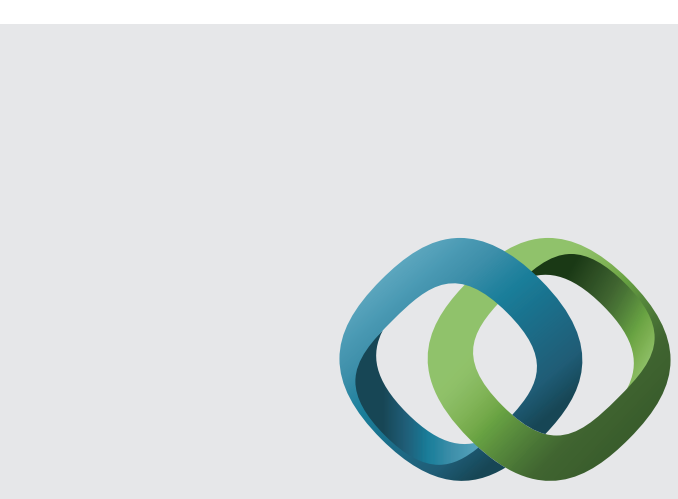

\section{Hindawi}

Submit your manuscripts at

http://www.hindawi.com
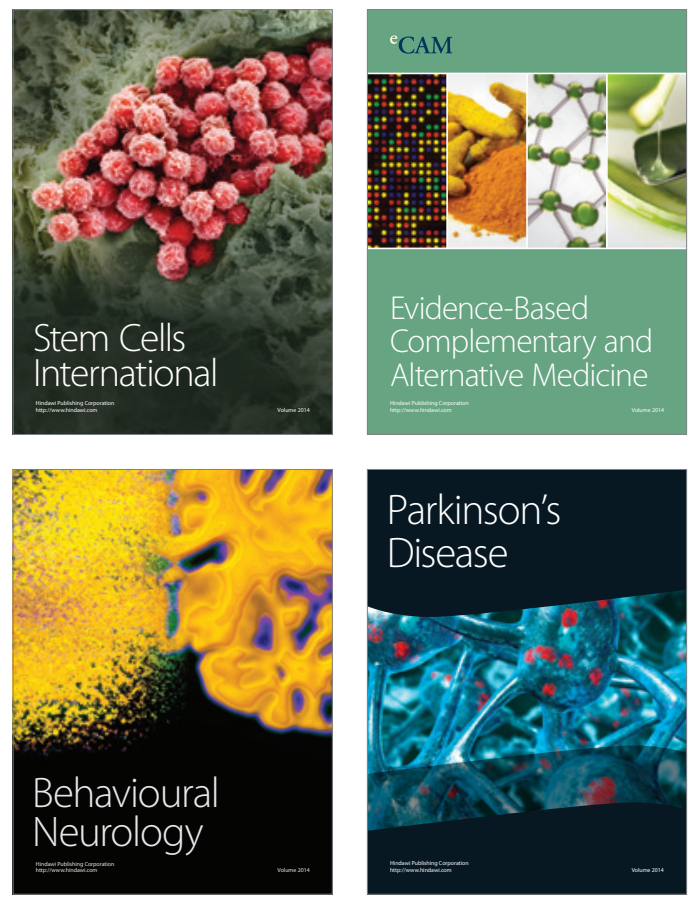
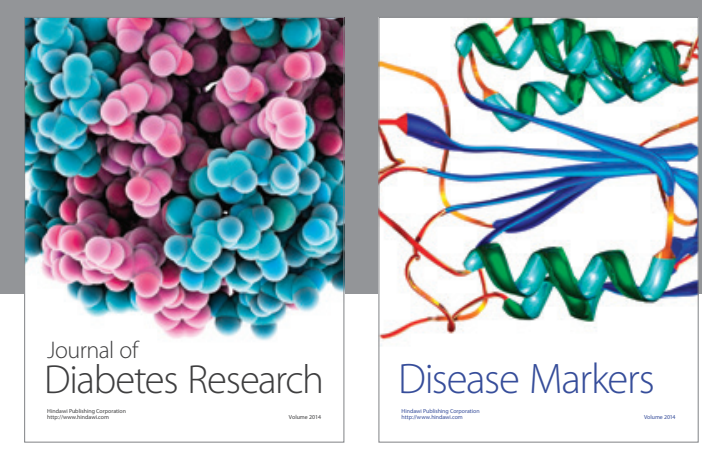

Disease Markers
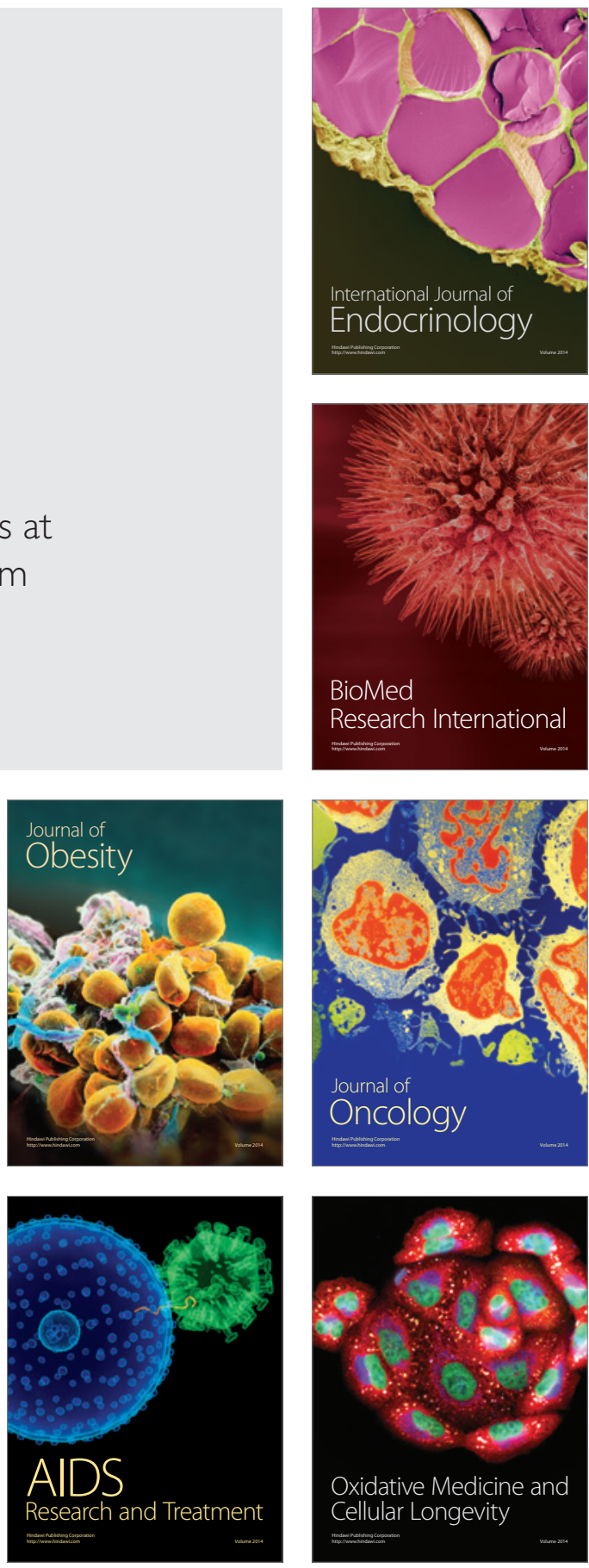